\title{
ON THE CONSTRUCTION OF SOLUTIONS OF GENERAL LINEAR BOUNDARY VALUE PROBLEMS FOR SYSTEMS OF FUNCTIONAL DIFFERENTIAL EQUATIONS
}

\author{
VERONIKA NOVOTNÁ AND BEDŘICH PƯŽA
}

Received 16 April, 2018

Abstract. For the linear boundary value problem

$$
x^{\prime}(t)=p(x)(t)+q(t), \quad l(x)=c_{0}
$$

on the closed interval $I \subset R$, where $p: C\left(I, R^{n}\right) \rightarrow L\left(I, R^{n}\right)$ is a strongly bounded linear operator, $l: C\left(I, R^{n}\right) \rightarrow R^{n}$ is the bounded linear functional, $q \in L\left(I, R^{n}\right)$ and $c_{0} \in R^{n}$, we describe a method of construction of its solution using successive approximations by the sequence of the solutions of simple boundary value problems. We prove the conditions which guarantee the convergence of the above mentioned sequences in general and special cases, we prove the stability of the convergence in some sense. Also, for illustration, we solve some typical problems in MAPLE.

2010 Mathematics Subject Classification: 34K06; 34K13; 34B15

Keywords: system of functional differential equations, general boundary value problems, argument deviation, construction of solutions, successive approximations

\section{STATEMENT OF THE MAIN RESULTS}

\subsection{Introduction}

Consider the system of linear functional differential equations on interval $I=$ $[a, b]$

$$
x^{\prime}(t)=p(x)(t)+q(t),
$$

with the linear boundary value conditions

$$
l(x)=c_{0},
$$

where $p: C\left(I ; R^{n}\right) \rightarrow L\left(I ; R^{n}\right)$ is a linear strongly bounded operator (i.e., $p$ is a linear operator and there is Lebesgue integrable function $\eta: I \rightarrow R_{+}$such that $\|p(x)(t)\| \leq \eta(t)\|x\|_{C}$ for almost all $\left.t \in I, x \in C\left(I ; R^{n}\right)\right), l: C\left(I ; R^{n}\right) \rightarrow R^{n}$ is a linear bounded functional (i.e., $l$ is linear functional and there is the constant $\alpha \in R_{+}$ such that $\|l(x)\| \leq \alpha\|x\|$ for all $\left.x \in C\left(I ; R^{n}\right)\right), q \in L\left(I ; R^{n}\right)$ and $c_{0} \in R^{n}$. 
The a solution of the problem (1.1), (1.2) is understood to be an absolutely continuous vector valued function $x: I \rightarrow R^{n}$ which satisfies the system (1.1) almost everywhere on $I$, and satisfies the conditions (1.2).

Papers dealing with boundary value problems contain numerous interesting and, in a sense, optimal conditions ensuring the unique solvability and correctness of various boundary value problems for linear functional differential equations and systems, both in regular and singular cases (see, e.g., [1-14,21,22] and references therein). However, unlike systems of ordinary differential equations, in the case of boundary value problems for more general systems of functional differential equations, there are still almost no methods for approximate construction of their solutions. We can mention in this relation the parametrization methods (see, e.g., [18, 24]) using the ideas close to $[17,19,20,23]$ in the ordinary case.

This lack is dealt with in the present paper, which gives a construction that can be considered as a generalization of a number of previously published. A special attention should be paid to work [12], to which this paper is a follow-up. The work [11], using the Fredholm problem (1.1), (1.2), provide general sufficient and, in the cases where $p$ is a Volterra type operator, also necessary and sufficient (in a sense, unimprovable) conditions for the unique solvability of the boundary value problems (1.1), (1.2). A number of consequences for specific kinds of systems (1.1) and special cases of boundary conditions (1.2) are given. The works mentioned also contain statements on the continuous dependence of solutions of problems in question on small changes in right-hand side terms of systems (1.1) and boundary conditions (1.2) (the so-called correctness of problems (1.1), (1.2)).

\subsection{Notation and definitions}

The following notations are used throughout the paper:

$R=]-\infty,+\infty\left[, \quad R_{+}=\left[0, \infty\left[; \chi_{I}\right.\right.\right.$ is a characteristic function of interval $I$, i.e.,

$$
\chi_{I}(t)= \begin{cases}1 & \text { for } t \in I \\ 0 & \text { for } t \notin I\end{cases}
$$

$R^{n}$ is the space of $n$-dimensional column vectors $x=\left(x_{i}\right)_{i=1}^{n}$ with elements $x_{i} \in R$ $(i=1, \ldots, n)$, and norm

$$
\|x\|=\sum_{i=1}^{n}\left|x_{i}\right|
$$

$R^{n \times n}$ is the space of $n \times n$-matrices $X=\left(x_{i k}\right)_{i, k=1}^{n}$ with elements $x_{i k} \in R(i, k=$ $1, \ldots, n$,$) and norm$

$$
\begin{gathered}
\|X\|=\sum_{i, k=1}^{n}\left|x_{i k}\right| \\
R_{+}^{n}=\left\{\left(x_{i}\right)_{i=1}^{n} \in R^{n}: x_{i} \geq 0(i=1, \ldots, n)\right\}
\end{gathered}
$$




$$
R_{+}^{n \times n}=\left\{\left(x_{i k}\right)_{i, k=1}^{n} \in R^{n \times n}: x_{i k} \geq 0(i, k=1, \ldots, n)\right\} ;
$$

if $x, y \in R^{n}$ and $X, Y \in R^{n \times n}$, then

$$
x \leq y \Leftrightarrow y-x \in R_{+}^{n}, X \leq Y \Leftrightarrow Y-X \in R_{+}^{n \times n} ;
$$

if $x=\left(x_{i}\right)_{i=1}^{n} \in R^{n}$ and $X=\left(x_{i k}\right)_{i, k=1}^{n} \in R^{n \times n}$, then

$$
|x|=\left(\left|x_{i}\right|\right)_{i=1}^{n},|X|=\left(\left|x_{i k}\right|\right)_{i, k=1}^{n} \text {; }
$$

$\operatorname{det}(X)$ is the determinant of matrix $X$;

$X^{-1}$ is the inverse matrix to $X$;

$r(X)$ is the spectral radius of matrix $X$;

$E$ is the unit matrix;

$\Theta$ is the zero matrix;

$C\left(I ; R^{n}\right)$ is the space of continuous vector functions ${ }^{1} x: I \rightarrow R^{n}$ with norm

$$
\|x\|_{C}=\max \{\|x(t)\|: t \in I\} ;
$$

if $x=\left(x_{i}\right)_{i=1}^{n} \in C\left(I ; R^{n}\right)$, then

$$
|x|_{C}=\left(\left\|x_{i}\right\|_{C}\right)_{i=1}^{n} ;
$$

$\tilde{C}\left(I ; R^{n}\right)$ is the space of absolutely continuous vector functions $x: I \rightarrow R^{n}$ with norm

$$
\|x\|_{\tilde{C}}=\|x\|_{C}+\left\|x^{\prime}\right\|_{L}
$$

$C\left(I ; R^{n \times n}\right)$ is the space of continuous matrix functions $X \rightarrow R^{n \times n}$;

$L\left(I ; R^{n}\right)$ is the space of vector valued functions $x: I \rightarrow R^{n}$ with the Lebesgue integrable elements, with norm

$$
\|x\|_{L}=\int_{a}^{b}\|x(t)\| d t
$$

if $x=\left(x_{i}\right)_{i=1}^{n} \in L\left(I ; R^{n}\right)$, then

$$
|x|_{L}=\left(\left\|x_{i}\right\|_{L}\right)_{i=1}^{n} ;
$$

$L\left(I ; R^{n \times n}\right)$ is the space of Lebesgue integrable matrix functions $X: I \rightarrow R^{n \times n}$;

If $g: C\left(I ; R^{n}\right) \rightarrow L\left(I ; R^{n}\right)$ is a linear operator, then $|g|: C\left(I ; R^{n}\right) \rightarrow L\left(I ; R^{n}\right)$ denotes a non-negative operator such that

$$
|g(x)| \leq|g|(|x|) \quad \text { for all } x \in C\left(I ; R^{n}\right) .
$$

If $Z \in C\left(I ; R^{n \times n}\right)$ is a matrix function with columns $z_{1}, \ldots, z_{n}$, and $g: C\left(I ; R^{n}\right) \rightarrow$ $L\left(I ; R^{n}\right)$ is a linear operator, respectively $g: C\left(I ; R^{n}\right) \rightarrow R^{n}$ is a linear functional, then $g(Z)$ denote a matrix function, respectively the constant matrix with columns $g\left(z_{1}\right), \ldots, g\left(z_{n}\right)$.

\footnotetext{
${ }^{1}$ A vector (matrix) function is said to be continuous, integrable, etc., if such are its elements.
} 
Let us say that $p: C\left(I ; R^{n}\right) \rightarrow L\left(I ; R^{n}\right)$ is the Volterra operator with respect to $t_{0} \in I$, if for any $t \in I$ and $x \in C\left(I, R^{n}\right)$ where $x(s)=0$ for $s \in I_{t_{0}, t}$ the equality $p(x)(s)=0$ holds for almost all $s \in I_{t_{0}, t}$, where

$$
I_{t_{0}, t}=\left\{\begin{array}{ll}
{\left[t_{0}, t\right]} & \text { if } t_{0} \leq t \\
{\left[t, t_{0}\right]} & \text { if } t<t_{0}
\end{array} .\right.
$$

Special cases of system frequently occurring in practice (1.1) are: a linear system of ordinary differential equations

$$
x^{\prime}(t)=P(t) x(t)+q(t)
$$

a linear system of ordinary differential equations with a deviated argument

$$
x^{\prime}(t)=P(t) x(\tau(t))+q(t),
$$

respectively with multiple argument deviations

$$
x^{\prime}(t)=\sum_{i=1}^{\bar{s}} P_{i}(t) x\left(\tau_{i}(t)\right)+q(t),
$$

where $P, P_{i} \in L\left(I, R^{n \times n}\right), \tau, \tau_{i}: I \rightarrow R$ are measurable functions $(i=1, \ldots, \bar{s})$ and if $\tau(t) \leq t, \tau_{i}(t) \leq t$ for almost all $t \in I$, then $\tau$ and $\tau_{i}$, are so-called delayed arguments, and $q \in L\left(I, R^{n}\right)$.

Systems (1.3), (1.4) and (1.5) are special cases of linear systems (1.1) with a Volterra operator.

For example special cases of conditions (1.2) are: the initial condition

$$
x\left(t_{0}\right)=c_{0},
$$

the multi-point boundary condition

$$
\sum_{j=1}^{s} A_{j} x\left(t_{j}\right)=c_{0},
$$

the Cauchy type condition

$$
x\left(\bar{t}_{0}\right)=l_{0}(x)+c_{0},
$$

and the periodic boundary condition

$$
x(b)-x(a)=0,
$$

where $\bar{t}_{0}, t_{j} \in I(j=1, \ldots, s), l_{0}: C\left(I, R^{n}\right) \rightarrow R^{n}$ is a linear bounded functional, $A_{j} \in R^{n \times n}(j=1, \ldots, s)$ and $c_{0} \in R^{n}$.

For the construction of solution of the problem (1.1), (1.2) and description of its properties we use the method and notifications from work [13]:

Let $x \in C\left(I ; R^{n}\right), k, m \in N$ and $t_{0} \in I$, then we use following notations:

$$
p^{0}(x)(t)=p^{k, 0}(x)(t)=x(t), \quad p^{k}(x)(t)=\int_{t_{0}}^{t} p\left(p^{k-1}(x)\right)(s) d s,
$$




$$
\Lambda_{k}=l\left(\sum_{i=1}^{k} p^{i-1}(E)\right), \hat{q}(t)=\int_{t_{0}}^{t} q(s) d s,
$$

and if $\Lambda_{k}$ is regular, then

$$
p^{k, m}(x)(t)=p^{m}(x)(t)-\left[\sum_{i=1}^{m} p^{i-1}(E)(t)\right] \Lambda_{k}^{-1} l\left(p^{k}(x)\right),
$$

and

$$
D^{k, m}\left(\hat{q}, c_{0}\right)(t)=\left[\sum_{i=1}^{k} p^{i-1}(E)(t)\right] \Lambda_{k}^{-1}\left[c_{0}-l\left(\sum_{i=1}^{k} p^{i-1}(\hat{q})\right)+\sum_{i=1}^{m} p^{i-1}(\hat{q})(t)\right] .
$$

\subsection{Main results}

The following fundamental propositions about the solvability of a general linear boundary value problem (1.1), (1.2) is proved in [13, Theorem 1.1.1]:

Proposition 1. The boundary value problem (1.1), (1.2) is uniquely solvable if the corresponding homogeneous problem

$$
\begin{aligned}
x^{\prime}(t) & =p(x)(t), \\
l(x) & =0
\end{aligned}
$$

has only the trivial solution.

Proposition 2. Let $k, m \in N, m_{0} \in N \cup\{0\}$ and $A \in R_{+}^{n \times n}$ be such that

$$
r(A)<1,
$$

$\Lambda_{k}$ is regular, and for all the solutions of homogeneous boundary value problem corresponding to the nonhomogeneous problem (1.1), (1.2), the inequality

$$
\left|p^{k, m}(x)\right|_{C} \leq A\left|p^{k, m_{0}}(x)\right|_{C}
$$

holds. Then problem (1.1), (1.2) is uniquely solvable.

It is clear that the solution of system (1.1) is also the solution of the systems

$$
\begin{aligned}
x(t) & =x\left(t_{0}\right)+\int_{t_{0}}^{t} p(x)(s) d s+\int_{t_{0}}^{t} q(s) d s=\ldots \\
& =p^{k}(x)(t)+\left[\sum_{i=1}^{k} p^{i-1}(E)(t)\right] x\left(t_{0}\right)+\left[\sum_{i=1}^{k} p^{i-1}(\hat{q})(t)\right]
\end{aligned}
$$


for any $k \in N$ and arbitrary value $x\left(t_{0}\right)$. If for our $k \in N$ the matrix $\Lambda_{k}$ is regular, then from the boundary condition (1.2) it follows that

$$
c_{0}=l\left(p^{k}(x)\right)+l\left(\sum_{i=1}^{k} p^{i-1}(E)\right) x\left(t_{0}\right)+l\left(\sum_{i=1}^{k} p^{i-1}(\hat{q})\right)
$$

and then

$$
x\left(t_{0}\right)=\Lambda_{k}^{-1}\left[c_{0}-l\left(p^{k}(x)\right)-l\left(\sum_{i=1}^{k} p^{i-1}(\hat{q})\right)\right] .
$$

Therefore, the solution of the problem (1.1), (1.2) is also the solution of equation

$$
x(t)=p^{k, m}(x)(t)+D^{k, m}\left(\hat{q}, c_{0}\right)(t) .
$$

and vice versa.

Let now $x_{0} \in C\left(I, R^{n}\right)$, and for arbitrary $v \in N$ the vector-function $x_{v}$ be defined by equality

$$
x_{v}(t)=p^{k, m}\left(x_{v-1}\right)(t)+D^{k, m}\left(\hat{q}, c_{0}\right)(t) .
$$

Then the next theorem is true.

Theorem 1. Let $k, m \in N, m_{0} \in N \cup\{0\}$, and $A \in R_{+}^{n \times n}$ be such that the matrix $\Lambda_{k}$ is regular,

$$
r(A)<1, \quad r\left(A|| p^{k, m_{0}}|(E)|_{C}\right)<1,
$$

and for arbitrary $x \in C\left(I, R^{n}\right)$ the inequality

$$
\left|p^{k, m}(x)\right|_{C} \leq A\left|p^{k, m_{0}}(x)\right|_{C}
$$

holds. Then the problem (1.1), (1.2) has unique solution $x$, and

$$
x=\lim _{\nu \rightarrow \infty} x_{v} \text { uniformly on I }
$$

where functions $x_{v}$ are defined by the equality (1.14) and $x_{0} \in C\left(I ; R^{n}\right)$ is arbitrary.

Proof. From the conditions of our theorem and Proposition 2 it follows that there is a unique solution $x$ of problem (1.1), (1.2). Now, let us assume that $x_{v}(v \in N)$ are vector functions defined by the equation (1.14) for arbitrary $x_{0} \in C\left(I ; R^{n}\right)$. Then from (1.13), (1.14), (1.16), and the positivity of the operator $\left|p^{k, m_{0}}\right|$, we obtain:

$$
\begin{aligned}
\left|x-x_{v}\right|_{C} & =\left|p^{k, m}\left(x-x_{v-1}\right)\right|_{C} \\
& \leq A\left|p^{k, m_{0}}\left(x-x_{\nu-1}\right)\right|_{C} \leq A|| p^{k, m_{0}}|(E)|_{C}\left|x-x_{v-1}\right|_{C} \\
& \leq\left(A|| p^{k, m_{0}}|(E)|_{C}\right)^{2}\left|x-x_{\nu-2}\right|_{C} \leq \ldots \\
& \leq\left(A \| p^{k, m_{0}}|(E)|_{C}\right)^{v}\left|x-x_{0}\right|_{C} .
\end{aligned}
$$


From conditions (1.15) it follows that

$$
\lim _{\nu \rightarrow \infty}\left(A|| p^{k, m_{0}}|(E)|_{C}\right)^{\nu}=\Theta,
$$

and from the last inequality, it is obvious that $x_{v} \rightarrow x$ uniformly on $I$, which prove our theorem.

Let us $p: C\left(I, R^{n}\right) \rightarrow L\left(I, R^{n}\right)$ the Volterra operator. Then, as it is proved in [13], the equality $p^{k, m_{0}}(x) \equiv x$ holds, and the conditions of Proposition 2 are sufficient and necessary for the unique solvability of problem (1.1), (1.2).

Theorem 2. Let $p$ be the Volterra operator with respect to $t_{0} \in I$. Then the boundary value problem (1.1), (1.2) is uniquely solvable if and only if there are $k, m \in N$ and $A \in R_{+}^{n \times n}$ such that the matrix $\Lambda_{k}$ is regular,

$$
r(A)<1,
$$

and for all $x \in C\left(I, R^{n}\right)$ the inequality

$$
\left|p^{k, m}(x)\right|_{C} \leq A|x|_{C}
$$

holds. Moreover, for the solution $x$ of the boundary value problem (1.1), (1.2), the representation $x=\lim _{v \rightarrow \infty} x_{v}$ holds, where functions $x_{v}(v \in N)$ are defined by equality (1.14), and $x_{0} \in C\left(I ; R^{n}\right)$ is arbitrary.

Proof. The first part of our theorem, the necessity and sufficiency of its conditions for unique solvability of problem (1.1), (1.2), when $p$ is the Volterra operator, follows from the theorems proved in [13].

On the other hand, from our assumptions it is clear that $p^{k, m_{0}}(x) \equiv x$ and then $\left|p^{k, m_{0}}\right|(E)=E$. Therefore, the assertion of the second part of our theorem concerning the construction of the solution follows from the second part of Theorem 1.

Remark 1. As problems (1.1), (1.2), and (1.13) are equivalent for such $k \in N$ that matrix $\Lambda_{k}$ is regular, problem (1.1), (1.2) is also equivalent to problem (1.1), (1.12).

The functional on the right-hand side of equality (1.12) is composed from continuous functionals and operators and it is continuous with respect to $x$. Therefore, in view of the unique solvability of problem (1.1), (1.2), we can construct the sequence $\left\{x_{v}\right\}_{v=1}^{\infty}$ of successive approximations for the solution of problem (1.1), (1.2) using the method of iterations, where

$$
x_{v}^{\prime}(t)=p\left(x_{v-1}\right)(t)+q(t)
$$

and

$$
x_{v}\left(t_{0}\right)=\Lambda_{k}^{-1}\left[c_{0}-l\left(p^{k}\left(x_{v-1}\right)\right)-l\left(\sum_{i=1}^{k} p^{i-1}(\hat{q})\right)\right],
$$

if $x_{0} \in C\left(I, R^{n}\right)$ is arbitrary. 
Remark 2. The above-mentioned method of successive approximations is in a certain sense stable. Let $x$ be the solution of problem (1.1), (1.2), and $\bar{x}_{v}$ be defined by equalities:

$$
\bar{x}_{v}(t)=p^{k, m}\left(\bar{x}_{v-1}\right)(t)+D^{k, m}\left(\hat{q}+\hat{\bar{q}}_{v}, c_{0}+\bar{c}_{v}\right)(t),
$$

where $\bar{q}_{\nu} \in L\left(I, R^{n}\right), \bar{c}_{\nu} \in R^{n}$ and

$$
\lim _{\nu \rightarrow \infty}\left(\int_{a}^{b}\left|\bar{q}_{\nu}(t)\right| d t+\left|\bar{c}_{\nu}\right|\right)=0,
$$

then

$$
\begin{aligned}
\left|x-\bar{x}_{v}\right|_{C} & \leq\left|p^{k, m}\left(x-\bar{x}_{v}\right)\right|_{C}+\left|D^{k, m}\left(\hat{\bar{q}}_{\nu}, \bar{c}_{v}\right)\right|_{c} \\
& \leq A^{v}\left|x-x_{0}\right|_{C}+\left|D^{k, m}\left(\hat{\bar{q}}_{\nu}, \bar{c}_{v}\right)\right|_{c} .
\end{aligned}
$$

From the last inequality, given our assumptions about $\bar{q}_{\nu}$ and $\bar{c}_{\nu}$, and the facts that $\lim _{v \rightarrow \infty} D^{k, m}\left(\hat{\bar{q}}_{v}, \bar{c}_{v}\right)=0$ (uniformly on $I$ ), and $A^{v} \rightarrow \Theta$ if $v \rightarrow \infty$, it follows that the sequence $\bar{x}_{v}$ converges to function $x$ uniformly on $I$. As the problems under consideration are equivalent, problem (1.19), (1.20) is also stable in this sense.

Remark 3. If we introduce notation $T(x)=p^{k, m}\left(x_{v-1}\right)(t)+D^{k, m}\left(\hat{q}, c_{0}\right)$, then $T: C\left(I, R^{n}\right) \rightarrow C\left(I, R^{n}\right)$ is a linear operator, and from inequality

$$
\begin{gathered}
|T(x)-T(y)|_{C}=\left|p^{k, m}(x-y)\right|_{C} \leq \\
\leq A \| p^{k, m_{0}}|(E)|_{C}|x-y|_{C},
\end{gathered}
$$

where $r\left(A|| p^{k, m_{0}}|(E)|_{C}\right)<1$, it follows that operator $T$ is a contraction.

It is clear that unique solvability of problem (1.1),(1.2), and possibility of construction of this solution by the method of successive approximations, follows from the Banach's fixed-point theorem.

The theorems stated above imply the following corollaries. First, if we assume that $l(x)=x\left(t_{0}\right)$, where $t_{0} \in I$ is arbitrary, we obtain the conditions of unique solvability and construction of the solution of the initial problem for a of linear functionaldifferential equations.

Corollary 1. Let $p$ be the Volterra operator. Then problem (1.1), (1.6) is uniquely solvable if there are $m \in N$, and $A \in R_{+}^{n \times}$, such that $r(A)<1$, and for arbitrary $x \in C\left(I, R^{n}\right)$ inequality

$$
\left|p^{m}(x)\right|_{C} \leq A|x|_{C}
$$

holds. Moreover, for solution $x$ of problem (1.1), (1.6) representation $x=\lim _{v \rightarrow \infty} x_{v}$ holds, where $x_{v}(v \in N)$ are the solutions of problem (1.19),

$$
x_{v}\left(t_{0}\right)=c_{0}
$$

and $x_{0} \in C\left(I, R^{n}\right)$ is arbitrary. 
If we assume that $p(x)(t)=P(t) x(\tau(t))$, and $l(x)=\sum_{j=1}^{s} A_{j} x\left(t_{j}\right)$, where $\tau$ : $I \rightarrow R$ is a measurable function, $\tau(t) \leq t$ for $t \in I, A_{j} \in R^{n \times n}$ and $t_{j} \in I$ if $j=$ $1, \ldots, s$ we obtain the conditions of unique solvability and construction of the solution of a multi-point problem for the system of linear differential equations with deviated argument.

Corollary 2. Let $\tau: I \rightarrow R$ be a measurable function, $\tau(t) \leq t$ for almost all $t \in I$, $u:]-\infty, a] \rightarrow R^{n}$ be a continuous bounded function, det $\Lambda_{1} \neq 0, x(t)=u(t)$ for $t \leq a$, and

$$
r\left(\int_{a}^{b} \bar{P}(t) d s+\sum_{1}^{s}\left|\Lambda_{1} A_{j}\right| \int_{a}^{t_{j}} \bar{P}(t) d t\right)<1,
$$

where $\Lambda_{1}=\sum_{j=1}^{s} A_{j}$, and $\bar{P}(t)=\chi_{I}(\tau(t))|P(t)|$. Then problem (1.4), (1.7) is uniquely solvable.

Moreover, for solution $x$ of problem (1.4), (1.7) the representation $x=\lim _{v \rightarrow \infty} x_{v}$ holds (uniformly on I), where $x_{v}(v \in N)$ are solutions of the problem

$$
\begin{aligned}
x_{v}^{\prime}(t) & =\chi_{I}(\tau(t)) P(t) x\left(\tau_{0}(t)\right)+\left(1-\chi_{I}(\tau(t))\right) P(t) u(\tau(t))+q(t), \\
x_{v}\left(t_{0}\right) & =\Lambda_{1}^{-1}\left[c_{0}-\sum_{j=1}^{s} A_{j} \int_{t_{0}}^{t_{j}}\left[\chi_{I}(\tau(t)) P(t) x_{v-1}(t)-q(t)\right) d t\right],
\end{aligned}
$$

where

$$
\tau_{0}(t)= \begin{cases}\tau(t) & \text { if } \tau(t) \geq a, \\ a & \text { if } \tau(t)<a,\end{cases}
$$

and $x_{0} \in C\left(I, R^{n}\right)$ is arbitrary.

Remark 4. An analogous proposition can be obtained also for the system with deviating arguments (1.5).

Remark 5. From Corollary 2, a criterion of unique solvability and description of successive approximations for the initial problem for system (1.4) with delay (see also Corollary 1) also follows. On the other hand Corollary 2 does not include a criterion of unique solvability and description of successive approximations for the periodic problem, because if $l(x)=x(b)-x(a)$, then $\Lambda_{1}=E-E=0$ and therefore $\operatorname{det} \Lambda_{1}=0$.

We will obtain a criterion of unique solvability and description of successive approximations for the periodic problem for the systems of linear differential equations with deviated arguments (1.4) and (1.5) assuming that $l(x)=x(b)-x(a)$ and $k=2$.

Corollary 3. Let $i=1, \ldots, \bar{s}$, the measurable functions $\tau_{i}: I \rightarrow R$ be such that $\tau_{i}(t) \leq t$ for almost all $t \in I$,

$$
\tau_{0 i}(t)= \begin{cases}\tau_{i}(t) & \text { if } \tau_{i}(t) \geq a \\ a & \text { if } \tau_{i}(t)<a\end{cases}
$$


$u:]-\infty, a] \rightarrow R^{n}$ be a continuous bounded function, and $P_{i} \in L\left(I, R^{n \times n}\right)$. Then if

$$
\operatorname{det} \Lambda_{2} \neq 0
$$

and

$$
r\left(\int_{a}^{b} \bar{P}(t) d t+\left|\Lambda_{2}^{-1}\right| \int_{a}^{b} \bar{P}(t) d t\right)<1
$$

where

$$
\Lambda_{2}=\int_{a}^{b} \sum_{i=1}^{\bar{s}} \chi\left(\tau_{i}(t)\right) P_{i}(t) d t, \quad \bar{P}(t)=\sum_{i=1}^{\bar{s}} \chi_{I}\left(\tau_{i}(t)\right)\left|P_{i}(t)\right|,
$$

system (1.5) has unique solution $x$ under the periodic condition

$$
x(b)-x(a)=c_{0}, \quad x(t)=u(t) \quad \text { for } t \in R \backslash I,
$$

and the representation $x=\lim _{v \rightarrow \infty} x_{v}$ holds, where $x_{v}(v \in N)$ are solutions of the

$$
\begin{aligned}
x_{\nu}^{\prime}(t)= & \sum_{i=1}^{\bar{s}} \chi_{I}\left(\tau_{i}(t)\right) P_{i}(t) x_{v-1}\left(\tau_{0 i}(t)\right)+ \\
& +\sum_{i=1}^{\bar{s}}\left(1-\chi_{I}\left(\tau_{i}(t)\right)\right) P_{i}(t) u(t)+q(t), \\
x_{\nu}(b)= & \Lambda_{2}^{-1}\left[c_{0}-\int_{a}^{b}\left(\sum_{i=1}^{\bar{s}} \chi_{I}\left(\tau_{i}(t)\right) P_{i}(t) x_{v-1}(t)-q(t)\right) d t\right]
\end{aligned}
$$

for arbitrary $x_{0} \in C\left(I, R^{n}\right)$.

If we assume that $l(x)=x\left(t_{0}\right)-l_{0}(x)$, we will obtain conditions of unique solvability and construction of the solution of Cauchy-Nicoletti type problems (1.1), (1.8) for arbitrary systems with the Volterra operator, with deviated arguments (1.4) and (1.5).

In the general case, we have

$$
\Lambda_{k}=E-\sum_{i=1}^{k} l_{0}\left(p^{i-1}(E)\right)
$$

and then from (1.12) we obtain

$$
x\left(t_{0}\right)=\Lambda_{k}^{-1}\left[c_{0}+l_{0}\left(p^{k}(x)\right)+\sum_{i=1}^{k} l_{0}\left(p^{i-1}(\hat{q})\right)\right] .
$$

Corollary 4. Let $k, m \in N, m_{0} \in N \cup\{0\}, A \in R_{+}^{n \times n}$ be such that the matrix $\Lambda_{k}$ from (1.22) be regular, and condition (1.17) holds, $u:]-\infty, a] \rightarrow R^{n}$ be a continuous bounded function, and for arbitrary $x \in C\left(I, R^{n}\right)$ inequality (1.18) holds. 
Then problem (1.1), (1.8) has unique solution $x$ and the representation $x=\lim _{v \rightarrow \infty} x_{v}$ holds, where $x_{v}(v \in N)$ are solutions of the problem (1.19),

$$
x_{v}\left(t_{0}\right)=\Lambda_{k}^{-1}\left[c_{0}+l_{0}\left(p^{k}\left(x_{v-1}\right)+\sum_{i=1}^{k} l_{0}\left(p^{i-1}\right)(\hat{q})\right]\right.
$$

for arbitrary $x_{0} \in C\left(I, R^{n}\right)$.

In this corollary if we assume that $l_{0}=0$, or $t_{0}=a, l_{0}(x)=x(b)$ or $t_{0}=b, l_{0}(x)=$ $x(a)$, we will obtain a criterion of unique solvability and construction of successive approximations for the periodic problems for the mentioned above systems.

Now, if we assume that $l_{0}(x) \equiv 0$, i,e., $x\left(t_{0}\right)=c_{0}$, then $\Lambda_{k}=E(k \in N), l_{0}(x)=$ $x(b), \bar{t}_{0}=a$, i.e., $x(a)=x(b)$, and therefore

$$
\begin{aligned}
& \Lambda_{1}=0, \\
& \Lambda_{2}=-\int_{a}^{b} p(E)(t) d t, \\
& \Lambda_{k}=-\sum_{i=1}^{k} \int_{a}^{b} p\left(p^{i-1}(E)\right)(t) d t \text { for } k \geq 3,
\end{aligned}
$$

and

$$
x\left(t_{0}\right)=\Lambda_{k}^{-1}\left[c_{0}+\int_{a}^{b} p\left(p^{k-1}(x)\right)(t) d t+\sum_{i=2}^{k} \int_{a}^{b} p\left(p^{i-2}(\hat{q})\right)(t) d t+\int_{a}^{b} q(s) d s\right] .
$$

Also, if $l_{0}(x)=x(a), \bar{t}_{0}=b$, i.e., $x(b)=x(a)$, then

$$
\begin{aligned}
& \Lambda_{1}=0 \\
& \Lambda_{2}=\int_{a}^{b} p(E)(t) d t \\
& \Lambda_{k}=\sum_{i=1}^{k} \int_{a}^{b} p\left(p^{i-1}(E)\right)(t) d t \text { for } k \geq 3,
\end{aligned}
$$

and

$$
x(b)=\Lambda_{k}^{-1}\left[c_{0}-\int_{a}^{b} p\left(p^{k-1}(x)\right)(t) d t-\sum_{i=2}^{k} \int_{a}^{b} p\left(p^{i-2}(\hat{q})\right)(t) d t-\int_{a}^{b} q(s) d s\right] .
$$

\section{EXAMPLES}

For the illustration of calculations of (converging to solution) approximations of solutions of a given problem, only such approximations are used, the graphs of which 
are differentiable under given conditions. On Figures 1 and 2 shown below, the function of "history" $u$ is marked by a dotted line $\cdots$, the "starting function" $x_{0}$ of the iterative process is marked by a dashed line - - - approximations of solutions are marked by a dot-and-dash line - - - , and the last calculated approximation of solution $x_{v}$ is marked by a solid line — . Analogous problems are solved by MAPLE in the works $[3,15,16]$.

2.1 .

Let us solve the problem

$$
x^{\prime}(t)=\lambda x(t)+\mu x(t-1), \quad x(0)=1,
$$

on interval $I=[0,10]$, where $u(t)=-t+1$ for $t \in[-1,0]$. The problem is solved by local methods in [2].

By choosing $\lambda=-3,5, \mu=4, x_{0}(t)=1$ and $v=9$ we obtain the 9th approximation of the solution of the problem, see Figure 1a.

By choosing $\lambda=0,5, \mu=-1, x_{0}(t)=1$ and $\nu=10$ we obtain the 10th approximation of the solution to the problem, see Figure $1 \mathrm{~b}$.

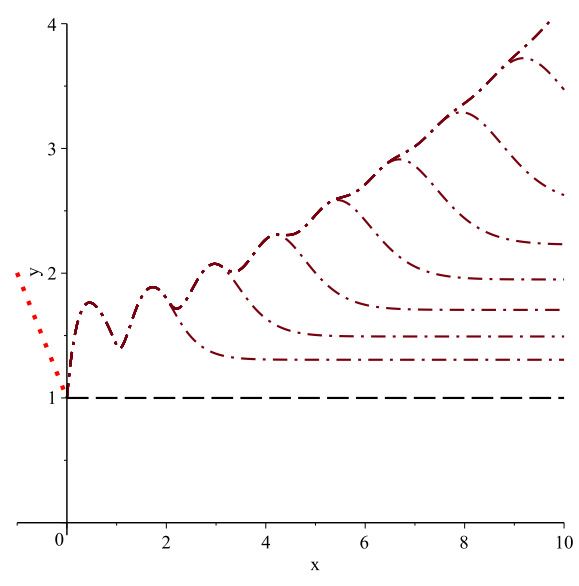

(a)

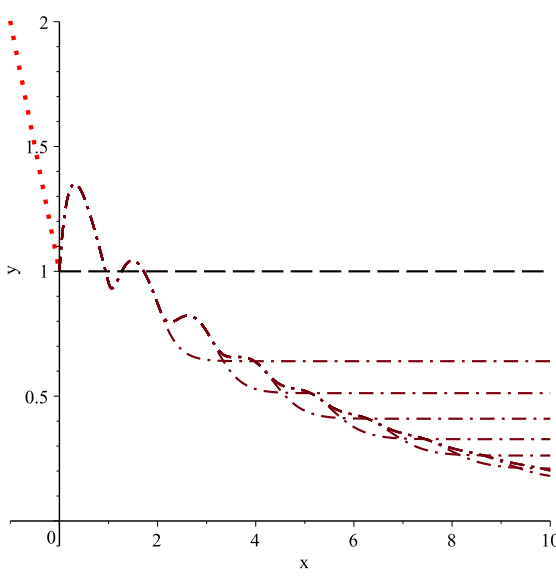

(b)

FIGURE 1. Solution of problem (2.1)

2.2.

Let us solve the problem

$$
\begin{aligned}
& x^{\prime}(t)=-x(t-1), \\
& x(0)=1,
\end{aligned}
$$

on interval $I=[0,3]$, where $x(t)=1$ for $t \in[-1,0]$. The problem is solved by the local methods in [2]. 
By choosing $x_{0}(t)=1$, we obtain the 3rd approximation of solution of the problem, see Figure 2a.

By choosing $x_{0}(t)=0$, we obtain the 3th approximation of solution to the problem, see Figure 2b, variant 2.

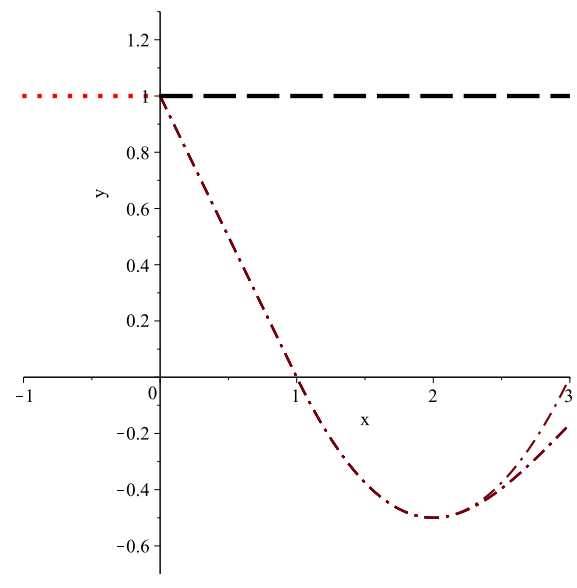

(a)

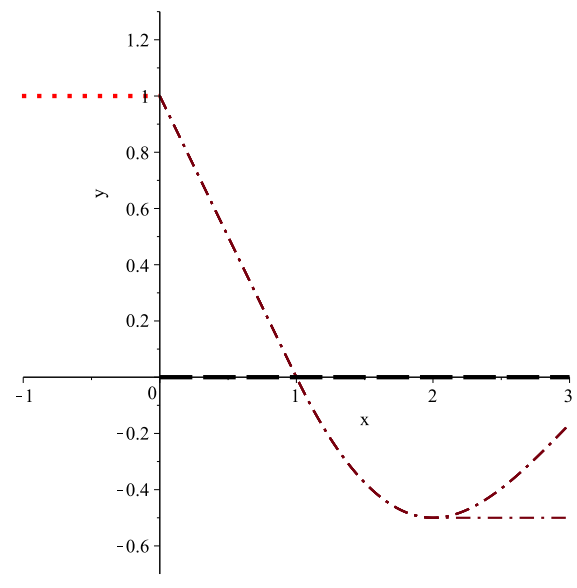

(b)

FIGURE 2. Solution of problem (2.2)

The next example is a generalization of the problem studied by Jan Tinbergen, a Dutch physicist and economist.

\section{3 .}

Let us solve the problem

$$
\begin{aligned}
f^{\prime}(t) & =-a(t) f(t-\delta(t)), \quad t \in[0, T], \\
f(t) & =h(t), \quad t \in\left[\Delta_{m}, 0\right],
\end{aligned}
$$

where functions $a$ and $\delta$ are (for the sake of simplification) continuous on interval $[0, T], \delta(t)>0$ for $t \in[0, T], \Delta_{m}=\min _{t \in[0, T]}(t-\delta(t))$, and $h$ is a continuous function on interval $\left[\Delta_{m}, 0\right]$. If $\delta(t) \equiv \Delta \geq 0$ (i.e., is a constant), equation (2.3) will be an equation with a constant delay and $\Delta_{m}=-\Delta$. Moreover, if $a(t) \equiv a>0$ i.e., (2.3) is a linear differential equation with constant coefficients and constant delay, studied by Jan Tinbergen. In this case we construct 6th $(v=6)$ approximation.

In order to demonstrate possibilities of a new approach to solution of the original problem, assume that the "historical development" prior to time $t=0$ can be simulated by the function $f=2 \sin t+1$ and that the length of delay will vary. For that reason, the paper [25] considered a situation in which the delay $\Delta(t)$ is not constant, but it is a function of time $t$. For illustration, we worked on the assumption that the 


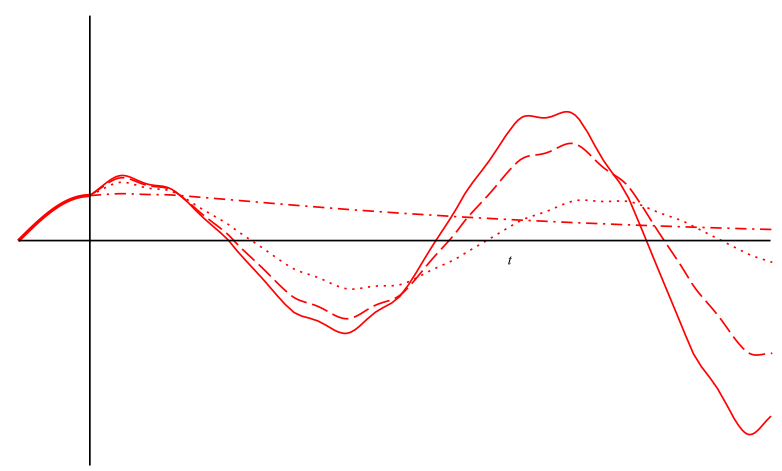

FIGURE 3. Solution to Tinbergen's model for a fluctuating delay

delay can be expressed by the function $\Delta(t)=2,5+0,5 \sin (5 t)$. The parameter $a$ was chosen in accordance with Tinbergen's findings.

\section{ACKNOWLEDGEMENT}

This paper was supported by the Czech Science Foundation, project No. GA1603796S.

\section{REFERENCES}

[1] N. V. Azbelev, V. P. Maksimov, and L. F. Rakhmatullina, Introduction to the Theory of Functional Differential Equations. Moscow: Nauka, 1991, in Russian, with an English summary.

[2] A. Bellen and M. Zennaro, Numerical methods for delay differential equations, ser. Numerical Mathematics and Scientific Computation. Oxford University Press, Oxford, 2013, first paperback reprint of the 2003 original [MR1997488].

[3] M. Bobalová and L. Maňásek, "On constructing a solution of a multipoint boundary value problem," in CDDE 2006: Colloquium on Differential and Difference Equations. Brno: Masaryk University, 2006, pp. 93-100.

[4] M. Bobalová and V. Novotná, "The use of functional differential equations in the model of the meat market with supply delay," Procedia - Social and Behavioral Sciences, vol. 213, pp. 74-79, 2015, 20th International Scientific Conference "Economics and Management 2015” ICEM-2015), doi: https://doi.org/10.1016/j.sbspro.2015.11.406. [Online]. Available: http://www.sciencedirect.com/science/article/pii/S1877042815057535

[5] E. Bravyi, "A note on the Fredholm property of boundary value problems for linear functional differential equations," Mem. Differential Equations Math. Phys., vol. 20, pp. 133-135, 2000.

[6] S. A. Brykalov, "Problems for functional-differential equations with monotone boundary conditions," Differential Equations, vol. 32, no. 6, pp. 740-747, 1997.

[7] N. Dilna and A. Ronto, "Unique solvability of a non-linear non-local boundary-value problem for systems of non-linear functional differential equations," Math. Slovaca, vol. 60, no. 3, pp. 327-338, 2010, doi: 10.2478/s12175-010-0015-9. [Online]. Available: https://doi.org/10.2478/s12175-010-0015-9

[8] N. Z. Dilnaya and A. N. Ronto, "Some new conditions for the solvability of the cauchy problem for systems of linear functional-differential equations," Ukrainian Math. J., vol. 56, 
no. 7, pp. 1033-1053, Jul 2004, doi: 10.1007/s11253-005-0068-z. [Online]. Available: https://doi.org/10.1007/s11253-005-0068-z

[9] S. Gelashvili and I. Kiguradze, "On multi-point boundary value problems for systems of functional-differential and difference equations," Mem. Differential Equations Math. Phys., vol. 5, pp. 1-113, 1995

[10] R. Hakl and S. Mukhigulashvili, "On a boundary value problem for $n$-th order linear functional differential systems," Georgian Math. J., vol. 12, no. 2, pp. 229-236, 2005.

[11] I. Kiguradze, An initial value problem and boundary value problems for systems of ordinary differential equations. Linear Theory. Tbilisi: Metsniereba, 1997, vol. 1, in Russian.

[12] I. T. Kiguradze, "Periodic solutions of systems of nonautonomous ordinary differential equations," Mat. Zametki, vol. 39, no. 4, pp. 562-575, 623, 1986, doi: 10.1007/BF01158003. [Online]. Available: https://doi.org/10.1007/BF01158003

[13] I. Kiguradze and B. Půža, "On boundary value problems for systems of linear functionaldifferential equations," Czechoslovak Math. J., vol. 47(122), no. 2, pp. 341-373, 1997, doi: 10.1023/A:1022829931363. [Online]. Available: https://doi.org/10.1023/A:1022829931363

[14] S. Mukhigulashvili, "Two-point boundary value problems for second order functional differential equations," Mem. Differential Equations Math. Phys., vol. 20, pp. 1-112, 2000.

[15] V. Novotná, "Numerical solution of the inventory balance delay differential equation," International Journal of Engineering Business Management, vol. 7, no. 1, pp. 1-9, 2015, doi: 10.5772/60113. [Online]. Available: https://doi.org/10.5772/60113

[16] V. Novotná and S. Škapa, "A nonlinear microeconomic model of goods production and sale using functional analysis," in The Role of Management in the Economic Paradigm of the XXIst Century. Bucharest: Bucharest University of Economic Studies, 2017, pp. 1-9.

[17] A. Rontó and M. Rontó, "Successive approximation techniques in non-linear boundary value problems for ordinary differential equations," in Handbook of differential equations: ordinary differential equations. Vol. IV, ser. Handb. Differ. Equ. Elsevier/North-Holland, Amsterdam, 2008, pp. 441-592.

[18] A. Rontó and M. Rontó, "On nonseparated three-point boundary value problems for linear functional differential equations," Abstr. Appl. Anal., pp. 1-22, Art. ID 326052, 2011, doi: 10.1155/2011/326052. [Online]. Available: http://dx.doi.org/10.1155/2011/326052

[19] A. Rontó, M. Rontó, and N. Shchobak, "Notes on interval halving procedure for periodic and two-point problems," Bound. Value Probl., pp. 2014:164, 20, 2014. [Online]. Available: https://doi.org/10.1186/s13661-014-0164-9

[20] A. Rontó, M. Rontó, and J. Varha, "A new approach to non-local boundary value problems for ordinary differential systems," Appl. Math. Comput., vol. 250, pp. 689-700, 2015. [Online]. Available: https://doi.org/10.1016/j.amc.2014.11.021

[21] A. Rontó and A. Samoilenko, "Unique solvability of some two-point boundary value problems for linear functional differential equations with singularities," Mem. Differential Equations Math. Phys., vol. 41, pp. 115-136, 2007.

[22] A. N. Ronto, "Exact conditions for the solvability of the Cauchy problem for systems of first-order linear functional-differential equations defined by $\left(\sigma_{1}, \sigma_{2}, \ldots, \sigma_{n} ; \tau\right)$-positive operators," Ukrainian Math. J., vol. 55, no. 11, pp. 1853-1884, 2003, doi: 10.1023/B:UKMA.0000027047.61698.48. [Online]. Available: https://doi.org/10.1023/B:UKMA.0000027047.61698.48

[23] A. Rontó, M. Rontó, and N. Shchobak, "Constructive analysis of periodic solutions with interval halving," Bound. Value Probl., pp. 2013:57, 34, 2013. [Online]. Available: https://doi.org/10.1186/1687-2770-2013-57

[24] A. Rontó, M. Rontó, and N. Shchobak, "On finding solutions of two-point boundary value problems for a class of non-linear functional differential systems," Electron. J. Qual. Theory Differ. Equ., no. 13, pp. 1-17, 2012. 
[25] T. Varyšová and V. Novotná, "Solution of dynamical macroeconomic model using modern methods," Journal of Eastern Europe Research in Business and Economics, no. 1, pp. 1-10, 2015, doi: 10.5171/2015.70142. [Online]. Available: https://doi.org/10.5171/2015.70142

\section{Authors' addresses}

\section{Veronika Novotná}

University of Technology, Faculty of Business and Management, Kolejni 4, 61200 Brno, Czech Republic

E-mail address: novotna@fbm.vutbr.cz

\section{Bedřich Půža}

Brno University of Technology, Faculty of Business and Management, Kolejni 4, 61200 Brno, Czech Republic

E-mail address: puza@fbm.vutbr.cz 